\title{
Outdoor exit detection using combined techniques to increase GPS efficiency
}

\author{
L.M. Soria Morillo, J.A. Ortega Ramírez, J.A. Alvarez García, L. Gonzalez-Abril
}

Keywords:

Location-dependent and sensitive Low-power design

Mobile environments

Pervasive computing

\begin{abstract}
The recent boom of GPS (Global Positioning System) as a universal method of location has meant that most people in developed countries have already used this technology sometime in their lives. However, this system suffers from an ever-increasing problem: energy expenditure. GPS receivers have been integrated into increasingly smaller devices such as the latest generation of mobiles, thereby making batterysaving a priority in the use of this technology. This article lays out a series of ideas which, through the use of auxiliary technologies, are able to maximize energy saving. By means of outdoor exit detection, it will be possible to automatically disconnect the GPS while the user stays indoors and later reconnect it on leaving the building.
\end{abstract}

\section{Introduction}

Nowadays, many companies have developed applications to control user activity for an extended time period by using ubiquitous systems. However devices use battery and they have a limited time of use, hence, the first requirement for the achievement of a ubiquitous system is to minimize the energy cost of the system. GPS receivers are embedded in last generation mobile phones and it is well-known that energy consumption when these devices are on is too high. This drawback makes the user experience with these systems are not quite beneficial. For this reason, several techniques that complement or replace the GPS system have been used in recent years, for instance the Wi-Fi technology which maintains very high precision in large cities and, in general, in highly populated urban areas. As can be seen in Fig. 1, APs density in urban places is very high (Torrens, 2011) and so, it is possible to base on these elements to carry out an indoor and outdoor positioning system. Thus, the Placelab tool (Letchner, Fox, \& LaMarca, 2005; Smith et al., 2005) is a tracking system based on wireless networks. The major advantage of this application is that no specific hardware is required. A similar system to Placelab was developed in Rekimoto, Miyaki, and Ishizawa (2007) in the Tokyo city, which deals with location through wireless networks where the density of these networks is spectacular. However, the drawback of these systems is accuracy since the detection is based on wireless networks and the accuracy would be compromised if the user resided in scarcely populated area or in a town far from any major city. Furthermore, even if the user is located in an area with high network density, the location is not necessarily accurate, since a geo- location network is needed so that approximate user positioning is possible. Thus, only in major cities can be possible to create a large enough database to obtain a reliable wireless network-based location. There are hardware alternatives that carry out geopositioning with lower battery drain (Raskovic \& Giessel, 2007; Tesoriero, Tebar, Gallud, Lozano, \& Penichet, 2010), but their greatest common disadvantages are the cost and also the use of specific devices that are difficult to integrate with elements present in daily lives. Thus, there are related systems based on dedicated hardware such that Keruve, Columba and SIMAP. The Keruve specific hardware attains a very effective tracking system for people with Alzheimer's disease based on location through GPS and GSM. The location accuracy problem is solved and the autonomy is very high since it is able to remain active for 3.5 days. Nevertheless, terminal use is not performed continuously, but on request and another problem is that communication between transmitter and receiver is made through telephone calls, which incurs a high cost for continuous positioning. Columba is another device used for elderly positioning. In this case, Columba alerts to users when the subject leaves the security zone, this is the space that surround an elderly person's house and only in this situation the GPS is enabled. For this reason, the autonomy of Columba system is slightly larger than the Keruve. However there is a drawback in Columba, this is so the system cannot position to its subject in each moment, but only when the user leaves at the security zone. Finally, SIMAP is a huge Spanish project that allows controlling users' activity and position. The main advantage of this system is the batteries autonomy (up to $52 \mathrm{~h}$ ) and a high level of customizing, due to allow configure safe areas, dangerous areas and integrate an accelerometer to detect falls and long inactivity period. However, the main drawback of this product is that users must carry a specific device, then the forgetting possibility is higher than when the logic is kept in the own mobile phone. There are studies whose target is to increase battery 


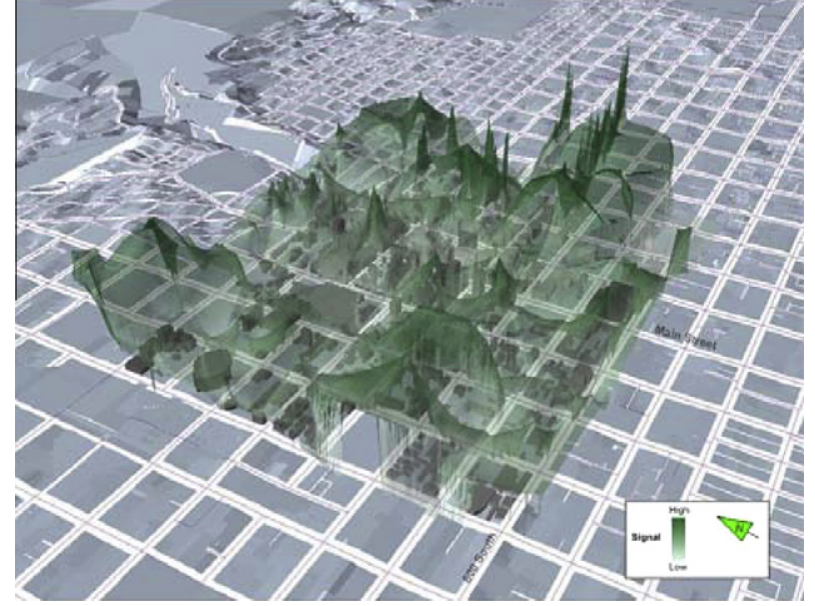

Fig. 1. Wi-Fi AP density on central Salt Lake City.

lifetime by using either less expensive sensors or by using more expensive sensors less frequently (Yim, 2008). In Ben Abdesslem, Phillips, and Henderson (2009), a system based on accelerometry is developed in order to save battery energy during the GPS positioning process. This system uses the cheapest sensor, that is, an accelerometer, to detect when a user is not moving and consequently switches off the GPS device to save energy. In the test process, results were very satisfactory as battery lifetime rose from 9 to $22 \mathrm{~h}$. However there are some situations in which users are walking indoors, and if the previous system was used then GPS would be enabled at all times and spending batteries. An example of this situation is if a teacher is at the chalkboard. Obviously the teacher is indoors, but he/she is moving, then GPS must not be activated. By taking advantages of the technologies integrated in the mobile phones, a new ubiquitous positioning system is proposed in this paper. This system combines GPS, Wi-Fi (Mengual, Marbán, \& Eibe, 2010; Yim, Jeong, Gwon, \& Joo, 2010) and accelerometry, and it could be designed for any mobile device, so the owner hardware problem would be solved. The scalability is a point in favor of the proposed system since general-purpose devices are used and there are no obstacles to adding new functions to the system. It is worth noting that the system is self-governing and it is not necessary that the user remains responsible for activating and deactivating the system as in the actual systems. This is the main concept of ubiquitous computing: users must be technologically-independent. With the system described in this paper the problems posed by the battery life in applications such as social networks or geolocation applications over the Internet are avoided. Thus the benefits of the technique developed could be applied to a vast number of applications which are increasingly making use of a ubiquitous GPS positioning system. In short, the problem of energy consumption is studied in an innovative way. The main objective is to determine when the user has entered indoors and, more importantly, to detect when the user leaves. Thus, it would be possible to disconnect the GPS device for as long as the user remains in an enclosed area without GPS coverage, and then to reconnect it on exit. This is highly significant if it is borne in mind that most GPS consumption occurs when the system tries to obtain reconnection to the signal from satellites. The remainder of the paper is organized as follows. Section 2 describes the different techniques used in the development of the system under study. In Section 3, these techniques are explained in depth, and in Section 4 the results are set out and compared in order to determine the best technique. Section 5 presents the future works identified based on this proposal and conclusions are drawn in Section 6.

\section{Energy efficiency comparison of technologies}

A comparison among the energy consumption of the Wireless (Wi-Fi networks), GSM Network, Accelerometry and GPS is carried out (Carroll \& Heiser, 2010; Zhang et al., 2010). This is critical in choosing the best outdoor detection method since, together with performance; these are the two main issues on which the final decision is based. First, the graphs only collect the hardware consumption associated to various techniques without any detection software coming into play. This decision is due to the fact that background software is common to all technologies, except certain modifications made for the adaption of each specific technology. Thus, with a constant cost for all methods, the software energy cost can be dismissed. All comparisons have been implemented in a Nexus S device with Android OS where all technologies are internally present, therefore the consumption of generic elements such as processor, memory and display are common to all methods and at no time has any additional hardware been used. All tests were carried out starting from an initial charge of $100 \%$ until the device shut down due to lack of battery. To avoid unnecessary memory access during the data collection process, this data has been collected only at these moments when the battery decreased by $10 \%$. For this reason, the energy consumption graph is staggered. Fig. 2 shows that the technology which consumes the most battery power is GPS. The average battery life using this system is about $7.5 \mathrm{~h}$, depending on satellite coverage. At the other extreme, there is the accelerometry sensor, since it works continuously in the device, and has no additional energy cost, except for the implementation of self-detection software. The battery lifetime period is approximately $60 \mathrm{~h}$ if the Wi-Fi, GPS, and GSM system are disconnected, hence it could be said that the approximate battery lifetime should match that of a totally unused device. However, the lifetime specified by the device is $130 \mathrm{~h}$, and this difference is due to background processes (such as $3 \mathrm{G}$ coverage, the operative system and running services) in addition to the software execution cost itself. At a point half-way between these two technologies the GSM and wireless methods are located. Regarding the GSM, the type of telephone coverage used by the device (GSM, 3G or UMTS) influences software energy cost. This cost is not due to mere data acquisition, given that the data used for positioning is obtained directly from the received signal, but by the additional cost incurred by the information being sent from the device to the telephone network and by the GSM valid signal search. With respect to the battery time using Wi-Fi networks indicates that the battery lasts barely $11 \mathrm{~h}$; a few hours more than when using the GPS device, although its duration is still fairly low. However, if detection by Wi-Fi networks were relied on for finding outdoor trips, a few hours of bat-

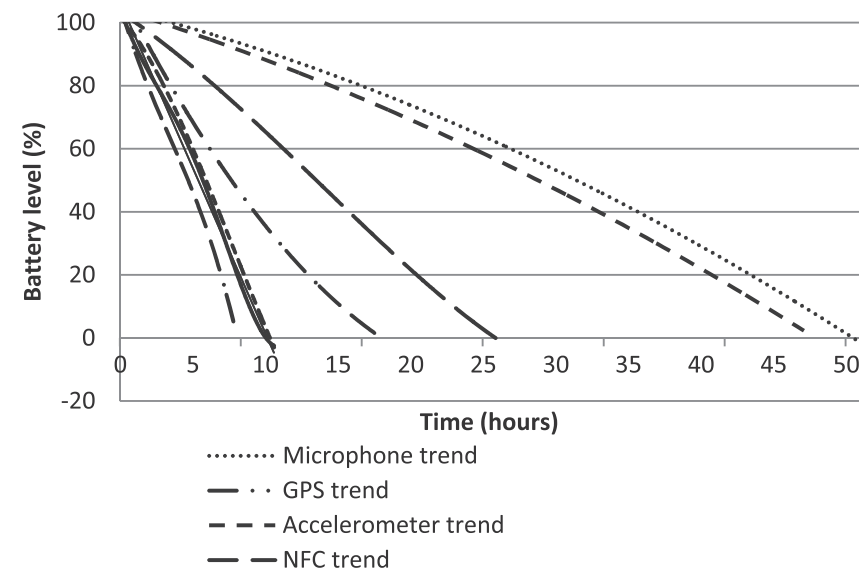

Fig. 2. Energy cost comparison between mobile sensors. 


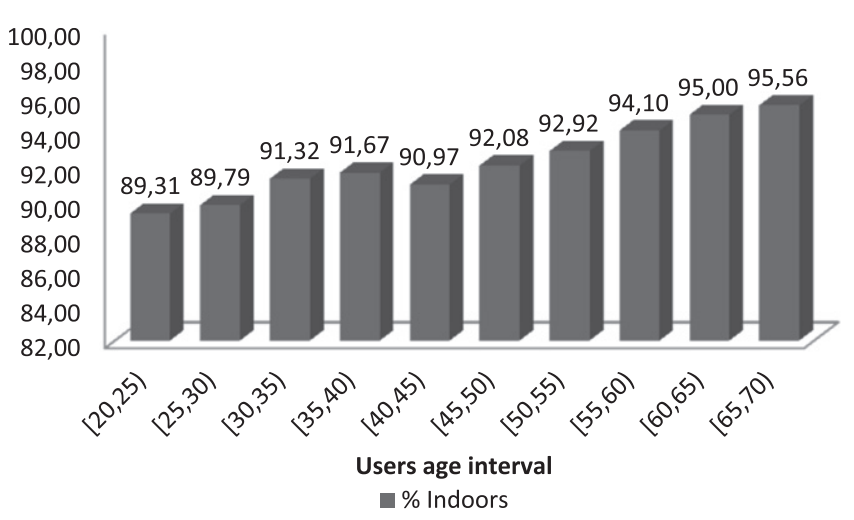

Fig. 3. Users time spent indoor per day studied through fifty users between 20 and 70 ages old.

tery would be gained that would be of great importance for this purpose: ubiquitous positioning. However, this technique, despite achieving successful implementation, provides no permanent solution since the cost in batteries is still very high for the purpose. An experiment has also been carried out to compute the average time a person remains indoors and outdoors throughout the day and hence conclusions about the efficiency of the system can be drawn. If the time a person stays outside is very high, the exit detection system is not going to have a very marked result on performance. However, if the time a person stays indoors is much greater than staying outside, then it is certain that it has a good impact on device energy consumption. To this end, a template was distributed to 50 different users upon which the results are calculated over two weeks. The results show that for an average of $91.2 \%$ of the total time, users remain in an enclosed environment, compared to $8.8 \%$ spent outdoors. Those results can be seen in Fig. 3. Through this study, it can be seen that users between 45 and 70 years old, the average is near to $94 \%$. That means that $22 \mathrm{~h}$ of each day those users remain indoors. Furthermore this fact is very important due to most of tracking and positioning systems are oriented to elderly people. Therefore, by using the above results together with those obtained in energy cost comparison of technologies, it can be said that by reducing the GPS use by $93 \%$ (the approximate average time of a user is outdoors) and by using auxiliary technologies in their place, about $70 \%$ of power consumption would be saved. Thus, if it combined the techniques that enable us to determine when the user has gone outside, together with the GPS positioning system, then a continuous location system is obtained whose energy consumption is sufficiently low to enable both normal mobile phone use and outdoor positioning for at least one full day.

\section{Building the outdoor exit detection system}

The moment of entry indoors needs to be detected in order to switch off the GPS and to avoid unnecessary cost, and also the moment of exit, in order to reactivate the GPS and accurately geolocation of the user. The moment of entry is simple to determine since prolonged loss of the GPS signal or low accurate positioning indicates that there is an obstacle that prevents receptor location. However, the second event is more complex to detect. To this end, a support system is required that does not consume too much energy. The following techniques are used:

- Detection based on Wi-Fi networks. The intensities of various Wi-Fi access points are recorded when entering a building, and similar intensities could be sought in order to discover when the user returns to the point of input, i.e. when the user leaves the building.
- Accelerometry. A movement is sought, and hence the patterns of movement are studied via the device that the user carries (Soria-Morillo, Álvarez Garćia, Ortega, \& González-Abril, 2010).

The aim of this work is not only to prevent high battery usage due to the GPS device, but also to do this in a way transparent to the user. In addition to everything described above, the system is able to detect movements of the user not only when walking, running or jumping, but also on all means of transport, for example, in the user's own car, by bus or train. No monitoring by the user is therefore required, which results in a ubiquitous system that increases energy efficiency of the device. Now that the basic ideas on the proposed system have been given, a clear distinction could be done between the different technologies employed.

\subsection{Based on Wi-Fi access points}

The outdoor detection system based on Wi-Fi access points consists of wireless network detection at the time when a GPS signal loss is detected. Once these networks are recorded, the period of the search for the moment of exit begins (Yun, Lee, Chung, Kim, \& Kim, 2009). This period consists of a constant detection of wireless networks available and a comparison of these with the networks recorded at the time that coverage was lost. When these two network sets match, the system come to the conclusion that the user is back to the starting point at which the signal was lost and therefore the outdoor exit is found. This process is not as easy as it seems, since the power fluctuation of the wireless signals makes the lower power signals appear and disappear from coverage in any particular place. For this reason a more advanced system must be developed and software must be made more accurate. First, as stated earlier, available wireless network points must be recorded. That is, by means of a search, a list of available networks with their associated signal strengths is obtained. This search is not performed only once, but several times and the sets of detected networks are saved. In this way, a set of searches is obtained which in turn contains a set of networks found with their signal strengths. The searches are carried out consecutively. Before the explanation of this method, must be said that Wi-Fi access points positions are not needed. In many works related with indoor positioning $\mathrm{Wi}-\mathrm{Fi}$ signals emitter position is essential (Lassabe, Canalda, Chatonnay, \& Spies, 2009), but this implies that if some signals are no geopositioned, these could not be used. In this work, all networks will be used, since the unique constraint is that can be accessed to. Thus, the information requirements needed for the development of a system based on wireless networks are the wireless networks identified by any unique parameter (MAC address) and information of signal intensity level measured in decibels. Using the BSSID (MAC address associated to an access point) as identifier and intensity as information, a Wi-Fi network can be written as:

wifiNet $=\left\{B S S I D, I_{B S S I D}\right\}$.

On the other hand, the network set found in each search, which consists of a set of all network information found at that place at a specific time $t$. This final set will be denoted hereafter as:

fingerprint $=\left\{\right.$ wifiNet $_{1}$, wifiNet $_{2}, \ldots$, wifiNet $\left._{m}\right\}$,

where $m$ is the number of Wi-Fi networks detected in each temporal window. Therefore, $m$ is not constant and could change between temporal windows if some networks have a low intensity or networks intensities are very fitful. For example, the first fingerprint is obtained, and before get the second one, the user crosses a door, then some networks which intensity is lower could disappear and networks number could change. Finally, a wireless window is defined between instants t 0 and th as the successive Wi-Fi traces detected at these moments and are denoted as: 
wifiWindow $=\left\{\right.$ fingerprint $_{0}$, fingerprint $_{1}, \ldots$, fingerprint $\left._{n}\right\}$.

In this case, $n$ is a constant and represents the number of windows that must be obtained to perform the matching exposed later. Once the network search process is finished, a statistical compilation is carried out designed to avoid the presence of false positive and false negative detections. As indicated earlier, the intensities of the networks (especially wireless home networks) are constantly changing. For this reason, the networks stored in the search process cannot be directly compared with existing networks; it is needed to go a step further. By obtaining the average power readings for each of the networks found in the search process, information on each of the networks is collected together with the range of fluctuation of its intensity values, in order to generate an automatic error margin depending on the characteristics of each network found. To explain this in a more formal way, let us look at two sets ( $n$ sets in general) formed by a number of elements (identifiers of the networks with the signal strength) all different in each of the sets. Once the sets are generated, common elements among them are sought, taking as a common element a pair of elements ( $n$ elements maximum in the case of $n$ sets) that possess the same ID (BSSID address). For each group of common elements, the following information is generated:

- Arithmetic mean of fingerprint $\left(I_{S S I D}\right)$.

- Standard deviation of $I_{S S I D}\left(S_{S S I D}\right)$.

Once these values for each network in the search sets are calculated, the results of both measures are stored since in the exitpoint finding process; these values are going to be needed for it to be identified. Another brief example of this system is taken. Suppose the application is configured to perform three searches. Thus the networks shown in Table 1 are obtained with the corresponding intensities. Each moment relates to the search that was conducted at that time. By calculating the statistics of each network, results shown in Table 2 are obtained.

Having generated all statistics for each network, an initial filter must be developed. This filter consists of the elimination of all sets of networks whose standard deviation is higher than the rest of the set. For this filtering it is necessary to put the set in ascending order of standard deviation. Once sorted, those sets with greater deviation are removed until the remaining number of sets is equal to $80 \%$ of all the initially existing sets, that is 2 of each 10 network sets are removed. This parameter was set to $80 \%$ in based on the application of the proposed method to a test set composed by $214 \mathrm{Wi}$-Fi networks, obtaining the best results for this value. Exit point detection is based on networks and stored statistical values, and their comparison with those of the networks detected at each instant. To determine whether a newly encountered network matches a stored network, the following similarity criteria should be met: the difference between the average power of the stored network and the found network power must be less than the

Table 1

Results of Wi-Fi search for three moments.

\begin{tabular}{lll}
\hline Search Moment & BSSID & $I_{B S S I D}(\mathrm{~dB})$ \\
\hline 1 & A2341f3F & -40 \\
& B112231F & -80 \\
& 11132323 & -20 \\
2 & A2341f3F & -30 \\
& B112231F & -90 \\
& 11132323 & -10 \\
& 23423424 & -30 \\
3 & A2341f3F & -40 \\
& B112231F & -80 \\
& 11132323 & -40 \\
\hline
\end{tabular}

Table 2

Wi-Fi search statistics for mean and standard deviation.

\begin{tabular}{lll}
\hline BSSID & $\left(I_{\text {BSSID }}\right)(\mathrm{dB})$ & $\left(S_{B S S I D}\right)(\mathrm{dB})$ \\
\hline A2341f3F & -37 & 4,72 \\
B112231F & -83 & 4,72 \\
11132323 & -23 & 12,47 \\
23423424 & -30 & 0 \\
\hline
\end{tabular}

network standard deviation at the time of the search. If exit detection algorithm finished here, then it would be concluded that the number of false negatives is very high. This is because the function for membership of encountered networks into the stored group is too restrictive. Some other parameter must be found to ease restrictions and make false negatives tend to disappear, since this situation could lead to an exit undetected by the device, for which the reception of the GPS signal would then fail to reactivate. This adaptation is implemented through the inclusion of a predefined error margin and a success percentage. The margin of error is used when determining network membership to the set of networks stored. The value is given in decibels and it creates a more permissive restriction than that mentioned earlier. Thus, a network belongs to the set of stored networks if the average powers obtained in the search process for that network minus the network strength at the time of detection is lower than the power network variation average at detection time multiplied by the established error margin.

Definition 1. A detected network is represented in the marked set provided if the following condition is observed:

$R_{j} \in C_{i} \leftrightarrow \frac{\left|\triangle I_{B S S I D}-\overline{I_{B S S I D}}\right|}{S_{B S S I D}}<\varepsilon$,

where $R_{j}$ is an access point detected in present time, $C_{i}$ is the set formed by all stored network instances same or most likely BSSID network address as $R_{j}$ in the Wi-Fi window generated in the exitmarking process and $\triangle I_{B S S I D}$ represents the intensity of $R_{j}$ reading.

The fact of dividing the subtraction between $\triangle I_{B S S I D}$ and $\overline{I_{B S S I D}}$ by the standard deviation is needed to provide a dynamic component to the exit detection system. In this case, if the access point detected presents a large deviation, error margin $(\varepsilon)$ must be greater than if the access point presents a smaller deviation. Instead modify the error margin, the subtraction is normalized based on standard deviation. In this way, the fluctuation problem of the wireless network power is reduced. Both the adaptive error margin (based on the average) and the constant error margin give very good results, as shown in the results section. After conducting a series of battery tests shown in Experiment section, it was concluded that the best $\varepsilon$ is 1.7. Despite having solved the problem of the variable intensity of networks, the problem still remains that weaker networks tend to disappear in the detection process, and a wireless network could even fail after the search process and hence, in the discovery process, this network would never be found. The network disappearing problem can be solved by using a success percentage. This percentage value indicates the number of networks in the search process that must match those stored to attain positive result detection.

Definition 2. A point will be interpreted as a departure point when the percentage of detected networks at that moment in time which meet the requirements of the membership function described above, is higher than the statically defined success percentage, this is:

isExitPoint $(C)=1 \leftrightarrow \frac{\sum_{i=0} \text { nbelongSet }\left(C_{i}\right)}{n}>\delta$. 
Wherein $\delta$ is the success percentage, and

$\operatorname{belongSet}\left(C_{i}\right)= \begin{cases}1, & \text { if } \exists R_{j} \in R / R_{j} \in C_{i}, \\ 0, & \text { otherwise }\end{cases}$

It has also been deduced, based on results and tests that are shown in Experiments section, that an appropriate value for the success rate is $\delta=0.7$, that is at least $70 \%$ of networks detected in the marking process at the exit point must match those identified at the present time. Both $\delta$ and $\varepsilon$ can be customized to the user environment of the exit detection system. For a general purpose, the values of $\varepsilon$ and $\delta$ are 1.7 and 0.7 respectively. However, they could be changed for achieve a higher precision if the user is in some special location. One of these locations is, for example, a village with few available Wi-Fi networks. In Tables 3 and 4, two Wi-Fi network detection examples and their classifications are shown. In the first example, three of the four detected networks meet the criteria that determine whether or not a network belongs to the stored network set, therefore the success percentage is $75 \%$. Given that the minimum success rate is defined as $70 \%$, can be said that the point where this network reading was detected coincides with the GPS loss point network. However, this does not hold true in the second example. Two of the four networks do not meet the criteria for membership, given that the deviation from the mean is higher than allowed, and hence the success rate is $50 \%$, which is less than the minimum for the point to be considered as an exit point. By examining the exit detection process based on Wi-Fi networks in a schematic form, a summary of the above explanation and generalization of the technique are obtained and represented in Fig. 4. The above algorithm gives very good results for exit detection, but the battery drain produced by the Wi-Fi networks searches has a huge impact over the system efficiency. When the user is moving, this search is necessary, but when users are stopped, the system efficiency could be improved if Wi-Fi search is halted. This solution is proposed in the next section, where accelerometry tools are used for detect user movement to turn on/off the Wi-Fi sensor.

\subsection{Based on accelerometry}

Currently, most mobile devices in the middle/upper range offer an accelerometer feature (Prekopcsák, 2008; Pylvänäinen, 2005), and can therefore be used for our purpose: to identify outdoor exits in order to reduce the consumption otherwise required for continuous localization. Data processing yields some characteristic values of the accelerometer measurement that can be employed to obtain a model that describes the activity according to these parameters. Of course, these parameters are not general but depend on the characteristics of each person who carries the device. The performance of this method is studied and compared with other methods already discussed. Although this is a technique that provides little information when the user is moving, it is very effective in practice since, in the majority of cases, most time is spent in the work place or at home, and in these places, where there is obviously no GPS coverage, the phone is commonly left on a desk or table. Therefore, this method is very effective in these cases, and the battery duration is greatly increased since during this time the GPS device is disabled. One problem that leads us to continue to seek other methods of detection is the inefficiency of accelerometry when movement occurs indoors. The method based on accelerometry reactivates the GPS signal when it detects a pattern of continuous movement by the user. But what if users moves indoors continuously? They have patterns of movement in their activities; however this pattern does not imply an exit. Thus, the GPS would be activated constantly in search of a valid signal which would never be found. Moreover, not only is the battery consumption affected by the fact that the GPS is always active, but the alternating pattern of movement and detention, also produces more on/off transitions in the GPS device, which increases battery consumption. In the Wi-Fi method and following methods, this problem is avoided, and a more robust system is created in these environments.

\subsection{Based on a combination of techniques}

As aforementioned, the use of techniques based on accelerometry and Wi-Fi pose a number of problems that are unsolvable when these techniques are used individually. To improve system efficiency it is necessary to join all the methods described above and create a unique method that combines the benefits of each one. This method is denoted here as the "Method based on a combination of techniques". The system structure involves:

- Accelerometry: it is used to detect user motion. This allows the system to disconnect other sensors when the device is immobile.

- Wi-Fi: it is used to mark the entrance point when the user enters indoors. It is also used to detect exits when the user crosses the en-trance point marked.

Fig. 5 clearly shows the states through which the system passes for the detection of exits from and entrances into places without GPS coverage using a combination of techniques. The algorithms

Table 3

Detection success rate for a reading set based on Table 2 with $\delta=0.7$ and $\varepsilon=1.7$.

\begin{tabular}{|c|c|c|c|c|}
\hline BSSID & $\triangle I_{B S S I D}$ & Deviation from the mean & Allowed deviation & Belong to set \\
\hline A2341f $3 F$ & $-40 \mathrm{~dB}$ & $3 \mathrm{~dB}$ & $8 \mathrm{~dB}$ & Yes \\
\hline B112231F & $-81 \mathrm{~dB}$ & $2 \mathrm{~dB}$ & $8 \mathrm{~dB}$ & Yes \\
\hline 11132323 & $-23 \mathrm{~dB}$ & $0 \mathrm{~dB}$ & $21.2 \mathrm{~dB}$ & Yes \\
\hline \multirow[t]{2}{*}{23423424} & $-32 \mathrm{~dB}$ & $2 \mathrm{~dB}$ & $0 \mathrm{~dB}$ & No \\
\hline & & $\delta=70 \%$ & $\varepsilon=1.7$ & Detected (75\%) \\
\hline
\end{tabular}

Table 4

Detection fail rate for a reading set based on Table 2 with $\delta=0.7$ and $\varepsilon=1.7$.

\begin{tabular}{|c|c|c|c|c|}
\hline BSSID & $\triangle I_{B S S I D}$ & Deviation from the mean & Allowed deviation & Belong to set \\
\hline $\mathrm{A} 2341 \mathrm{f} 3 \mathrm{~F}$ & $-47 \mathrm{~dB}$ & $10 \mathrm{~dB}$ & $8 \mathrm{~dB}$ & No \\
\hline B112231F & $-81 \mathrm{~dB}$ & $2 \mathrm{~dB}$ & $8 \mathrm{~dB}$ & Yes \\
\hline 11132323 & $-23 \mathrm{~dB}$ & $0 \mathrm{~dB}$ & $21.2 \mathrm{~dB}$ & Yes \\
\hline \multirow[t]{2}{*}{23423424} & $-32 \mathrm{~dB}$ & $2 \mathrm{~dB}$ & $0 \mathrm{~dB}$ & No \\
\hline & & $\delta=70 \%$ & $\varepsilon=1.7$ & Not detected (50\%) \\
\hline
\end{tabular}




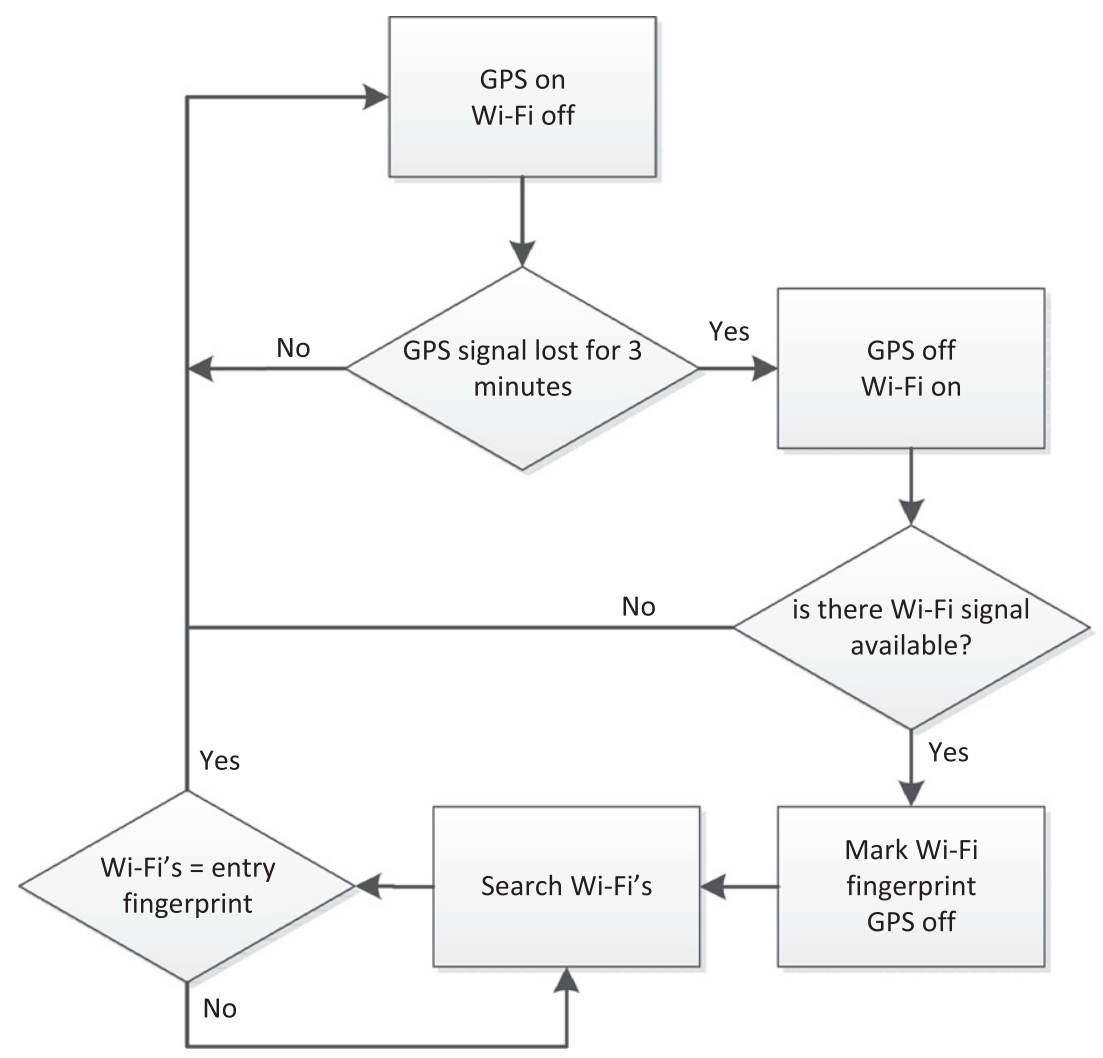

Fig. 4. Wi-Fi based outdoor detection schema.

used in this combined technique are the same as those used for the development of separate techniques. The only difference between this exit detection method and the others is that various techniques have been linked to increase detection efficiency and accuracy. Obviously, the schema for this case is more complex than previous methods, due to the transition between the three methods. In the first place, the possible entrance of a user is detected when the GPS signal is lost. On losing the signal, two alternatives exist depending on whether there is Wi-Fi coverage. If Wi-Fi networks exit where the user is, then the entrance point is marked and the GPS receiver is then disconnected. Otherwise, it is impossible to mark this point using this technique and the detection system based on the accelerometer is activated. When it detects that the user has stopped moving for a specified time period, the Wi-Fi sensor is disconnected if already activated, otherwise the GPS receiver is disconnected. Meanwhile, the system waits for a user movement using accelerometry. If is detected that the user is in motion, the Wi-Fi sensor is reactivated if the entrance point was marked previously.

\section{Experiments}

Now that the exit detection techniques have been explained in details an analysis of each technique can be performed to obtain results and the degree of reliability. Through this analysis two methods can be compared and that which involves a lower energy cost for the device and has greater accuracy can be chosen. 15 users have been chosen equipped with devices compatible with the application under development. Once the utility is installed in their devices, users are asked to perform their normal daily activity and to record on a sheet provided when they enter indoors and exit outdoors. This procedure was carried out for 20 days by each user. By comparing the application and the form filled in by each user, can be able to determine the percentage of correct answers given by the application for detection of entries and exits, and the number of errors, as well as the number of false positives (detection of a non-existent exit) and false negatives (failure to detect an exit). Regarding the categorization of errors, can be said that a reading is in error when an exit by the user remains undetected for more than $5 \mathrm{~min}$. With the results shown in Table 5, two different comparisons can be made in order to choose the best technique. One criterion is the technique accuracy, which is calculated based on the percentage of errors. On the other hand, a comparison of the battery life for each technique is made, since the aim of this study is to reduce the consumption of batteries in order to ensure continuous and effective monitoring with $\varepsilon$ and $\delta$ out any intervention by the user and with the greatest possible autonomy. Previous data have been obtained through an application developed for this purpose. The application has been developed for Android devices with 2.3 platforms at least. This application has been run on Nexus S devices powered by Google. These devices have been distributed to 15 users for test de proposed method. With this application has been possible to mark the Wi-Fi network surrounding the user and search matching with the Wi-Fi stored for detect de exit. Fig. 6a shown the main menu of the application, which allows beginning the process, search networks (advanced mode) and mark Wi-Fi networks manually (advanced mode). Fig. 6b shown the manually search process to identify the access points nearest to the device. If the decision is based on the technique accuracy, it can be seen that both techniques showed have a similar error rate. This is because the technique which uses the accelerometer is complementary to Wi-Fi technique. Then, the only effect of the accelerometer is a greater time of battery. This aim is achieved due to the Wi-Fi search is stopped when the system based on accelerometry detect that the user is not moving. Furthermore, another point taken into account when this exit detection system was developed was the energetic consumption. Knowing that the 


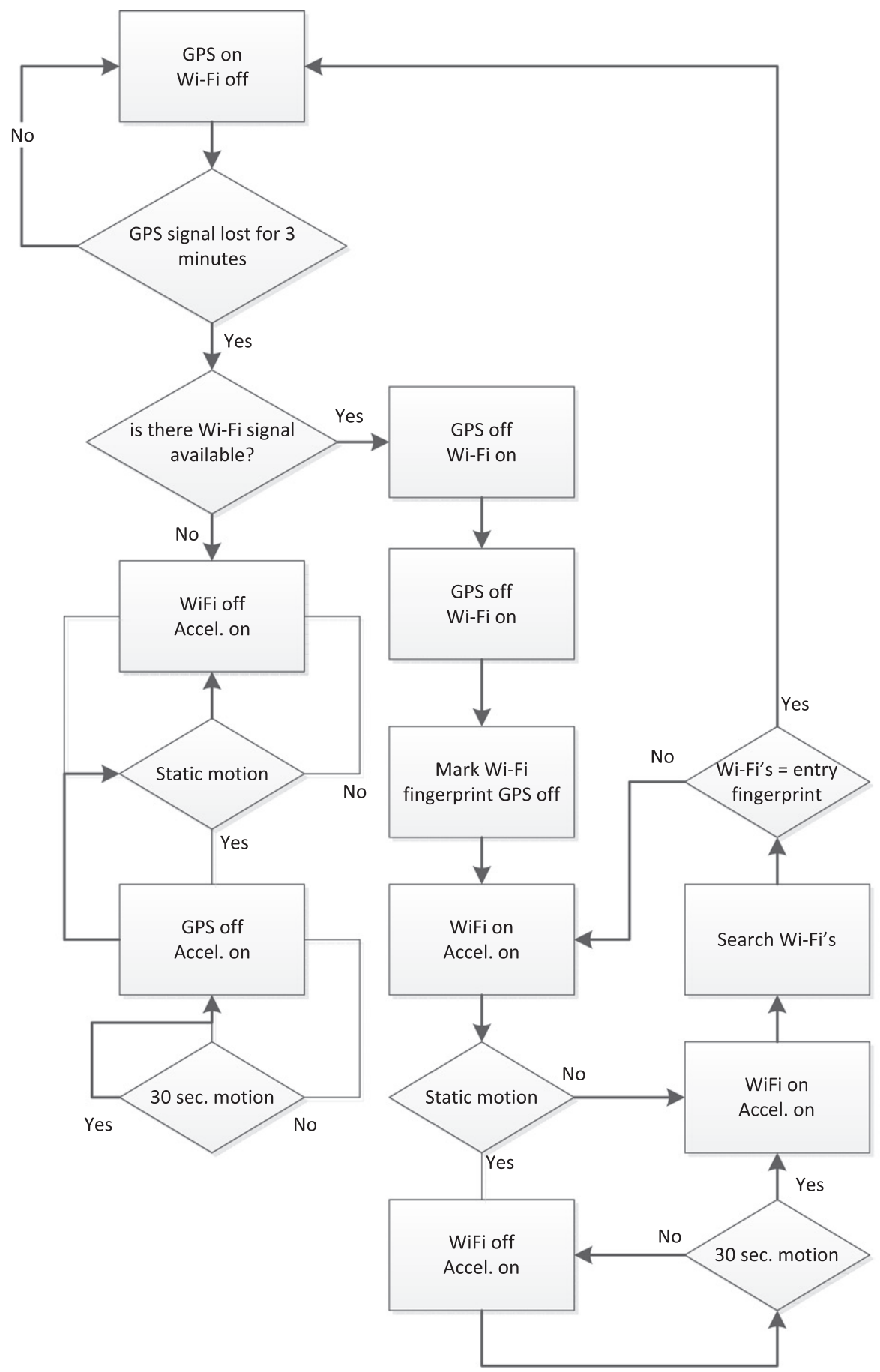

Fig. 5. Schema of combination techniques based outdoor detection.

developed system allows saving about $17 \mathrm{~h}$ for each recharge in continuous location systems, as was exposed previously, and taken into account that power factor in the smartphone used during the test process is $80 \%$ :

$P=V \cdot I=3^{\prime} 7 \mathrm{~V} \cdot 2^{\prime} 4 \mathrm{Ah} \cdot 0^{\prime} 8=7^{\prime} 1 \mathrm{~W}$,

$E_{\text {consumed }}=P \cdot \Delta t=7^{\prime} 1 \mathrm{Wh} \cdot 17 \mathrm{~h}=121 \mathrm{~W}$.

It is to say, for each recharge cycle the system could save $121 \mathrm{~W}$, or that it is the same $0^{\prime} 13 \mathrm{KW}$. Based on a study carried out by Nielsen Consulting in 2011, there are $18 \mathrm{M}$ of smartphones in Spain, $7 \mathrm{M}$ of them have GPS chipsets and, being pessimist, there 500.000 users that could be using the proposed method. In this case, by using
Table 5

Comparison of device battery life (in hours) and accuracy using both techniques implemented.

\begin{tabular}{llll}
\hline Technique & GPS & Wi-Fi & Wi-Fi + acc \\
\hline \% success & $100 \%$ & $95 \%$ & $94 \%$ \\
\% false positives & $95 \%$ & $3 \%$ & $3 \%$ \\
\% false negatives & $0 \%$ & $2 \%$ & $2 \%$ \\
\% total errors & $95 \%$ & $5 \%$ & $5 \%$ \\
Battery time (hours) & 8.2 & 16.5 & 25.8 \\
Number of transitions made & 630 & 570 & 728
\end{tabular}

the exposed system, we can save 39 tons of $\mathrm{CO}_{2}$, as can be seen in Table 6. 


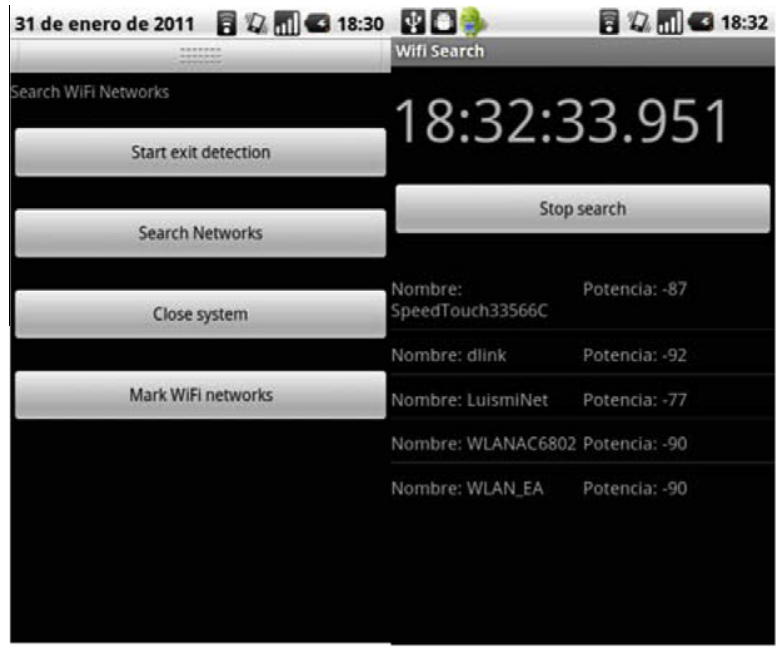

Fig. 6. (a) Main menu of the application developed to test de method proposed, (b) Manually Wi-Fi search process to obtain near Wi-Fi access points.

Table 6

Amount of $\mathrm{CO}_{2}$ not emitted to atmosphere by using the proposed system with 500.000 users.

\begin{tabular}{ll}
\hline Time & Amount of $\mathrm{CO}_{2}$ not emitted (Tons) \\
\hline Day & 39 \\
Week & 273 \\
Month & 1170 \\
Year & 14235 \\
\hline
\end{tabular}

\section{Future works}

The main drawback of this proposal is the case of the building where the user is, has two or more entry doors. In this way, the applications developed will recognize the exit if and only if the user performs the departure using the same door (or other very close to) that used to in. Once identified this problem, we are developing based on this technique, a new system for not only detect the exit, but mark different rooms or places inside a building and associate this places to some characteristics and obtain a semantic indoor positioning system. By this way, we could identify some areas in which are probable that users perform exits. Furthermore, by using an adaptive GPS frequency to obtain data, we are able to detect new exist even if the user never has carry out an entry or exit before.

\section{Conclusions}

The goal of this research was to develop a method to avoid the high energy consumption produced by GPS receivers, especially on mobile devices. Results section shows this has been achieved. The battery life of a GPS device in constant use is normally $7 \mathrm{~h}$. Thanks to combined technique based on Wi-Fi and accelerometry, the battery life can be increased to $25 \mathrm{~h}$. Therefore, mobile devices and especially applications that make constant use of GPS as a geopositioning technique could reduce their energy costs thanks to such technology. Consequently, battery life would not be the principal restricting factor, thus leaving the way clear for us to take full advantage of the use of applications based on user location in conjunction with the methods of interconnection available on mobile devices.

\section{Acknowledgments}

This research is supported by the Spanish Ministry of Science and Innovation R\&D project ARTEMISA (TIN2009-14378-C02-01).

\section{References}

Ben Abdesslem, F., Phillips, A., \& Henderson, T. (2009). Less is more: Energy-efficient mobile sensing with senseless. In Proceedings of the 1st ACM workshop on networking, systems, and applications for mobile handhelds. MobiHeld '09 (pp. 61-62). New York, USA: ACM. http://dx.doi.org/10.1145/1592606.1592621.

Carroll, A., \& Heiser, G. (2010). An analysis of power consumption in a smartphone. In Proceedings of the 2010 USENIX conference on USENIX annual technical conference. USENIXATC'10 (pp. 21). Berkeley, CA, USA: USENIX Association<http://dl.acm.org/citation.cfm?id=1855840.1855861>.

Lassabe, F., Canalda, P., Chatonnay, P., \& Spies, F. (2009). Indoor Wi-Fi positioning: Techniques and systems. Annals of Telecommunications, 64, 651-664. http:// dx.doi.org/10.1007/s12243-009-0122-1.

Letchner, J., Fox, D., \& LaMarca, A. (2005). Large-scale localization from wireless signal strength. In Proceedings of the 20th national conference on Artificial intelligence - Volume 1. AAAI'05 (pp. 15-20). AAAI Press<http://dl.acm.org/ citation.cfm?id=1619332.1619337>.

Mengual, L., Marbán, O., \& Eibe, S. (2010). Clustering-based location in wireless networks. Expert Systems with Applications, 37(9), 6165-6175<http:// www.sciencedirect.com/science/article/pii/S0957417410001508>.

Prekopcsák, Z. (2008). Accelerometer based real-time gesture recognition.

Pylvänäinen, T. (2005). Accelerometer based gesture recognition using continuous hmms. In J. Marques, N. Pérez de la Blanca, \& P. Pina (Eds.), Pattern recognition and image analysis. Lecture notes in computer science (Vol. 3522, pp. 413-430). Berlin, Heidelberg: Springer. http://dx.doi.org/10.1007/11492429_77.

Raskovic, D., \& Giessel, D. (2007). Battery-aware embedded GPS receiver node. In International conference on mobile and ubiquitous systems: Networking and services, 2007. MobiQuitous 2007 (pp. 1-6).

Rekimoto, J., Miyaki, T., \& Ishizawa, T. (2007). Lifetag: Wifi-based continuous location logging for life pattern analysis. In LoCA (pp. 35-49). <http:// www.sonycsl.co.jp/person/rekimoto/papers/loca07.pdf>.

Smith, I., Tabert, J., Wild, T., LaMarca, A., Chawathe, Y., Consolvo, S., et al. (2005). Placelab: Device positioning using radio beacons in the wild. In Proceedings of the third international conference on pervasive computing (pp. 116-133). Springer.

Soria-Morillo, L. M., Álvarez García, J. A., Ortega, J. A., \& González-Abril, L. (2010). Tracking system based on accelerometry for users with restricted physical activity. In Proceedings of the 23rd international conference on industrial engineering and other applications of applied intelligent systems - Volume part II. IEA/AIE'10 (pp. 470-483). Berlin, Heidelberg: Springer-Verlag<dl.acm.org/ citation.cfm?id=1945847.1945904>.

Tesoriero, R., Tebar, R., Gallud, J., Lozano, M., \& Penichet, V. (2010). Improving location awareness in indoor spaces using RFID technology. Expert Systems with Applications, 37(1), 894-898<http://www.sciencedirect.com/science/article/pii/ S095741740900503X>.

Torrens, P. (2011). Urban geosimulation. Agent-based models of geographical systems: Moving from theory to real world applications. Springer-Verlag.

Yim, J. (2008). Introducing a decision tree-based indoor positioning technique. Expert Systems with Applications, 34(2), 1296-1302 <http://www.sciencedirect. com/science/article/pii/S0957417406004179>.

Yim, J., Jeong, S., Gwon, K., \& Joo, J. (2010). Improvement of Kalman filters for Wlan based indoor tracking. Expert Systems with Applications, 37(1), 426-433<http:// www.sciencedirect.com/science/article/pii/S0957417409004916>.

Yun, S., Lee, J., Chung, W., Kim, E., \& Kim, S. (2009). A soft computing approach to localization in wireless sensor networks. Expert Systems with Applications, 36(4), 7552-7561 <http://www.sciencedirect.com/science/article/pii/S095741 7408006830>.

Zhang, L., Tiwana, B., Qian, Z., Wang, Z., Dick, R. P., Mao, Z. M., \& Yang, L. (2010). Accurate online power estimation and automatic battery behavior based power model generation for smartphones. In Proceedings of the eighth IEEE/ACM/IFIP international conference on hardware/software codesign and system synthesis. CODES/ISSS '10 (pp. 105-114). New York, USA: ACM. http://dx.doi.org/10.1145/ 1878961.1878982 